General Mathematics Vol. 28, No. 1 (2020), 125-140

DOI: $10.2478 / \mathrm{gm}-2020-0010$

S sciendo

\title{
Certain subclasses of univalent and bi-univalent functions related to shell-like curves connected with Fibonacci numbers ${ }^{1}$
}

\author{
Gurmeet Singh, Gagandeep Singh, Gurcharanjit Singh
}

\begin{abstract}
This paper is concerned with certain subclasses of univalent and bi-univalent functions related to shell-like curves connected with Fibonacci numbers. We find estimates of the initial coefficients $\left|a_{2}\right|$ and $\left|a_{3}\right|$ for the functions in these classes. Also we investigate upper bounds for the Fekete-Szegö functional and second Hankel determinant for these classes.
\end{abstract}

2010 Mathematics Subject Classification: 30C45, 30C50.

Key words and phrases: Sãlãgean operator, bi-univalent functions, subordination, shell-like curves, univalent functions, starlike functions, convex functions, Fibonacci numbers.

\section{Introduction}

Let $A$ be the class of functions of the form

$$
f(z)=z+\sum_{k=2}^{\infty} a_{k} z^{k}
$$

which are analytic in the unit disc $U=\{z:|z|<1\}$ with normalization $f(0)=$ $f^{\prime}(0)-1=0$. By $S$, we denote the class of functions $f(z) \in A$ and univalent in $U$.

\footnotetext{
${ }^{1}$ Received 2 March, 2019

Accepted for publication (in revised form) 29 January, 2020
} 
Let us denote by $B$, the class of bounded or Schwarz functions $w(z)$ satisfying $w(0)=0$ and $|w(z)| \leq 1$ which are analytic in the open unit disc $U$ and of the form

$$
w(z)=\sum_{n=1}^{\infty} c_{n} z^{n}, z \in U .
$$

Consider two functions $f$ and $g$ analytic in $U$. We say that $f$ is subordinate to $g$ (symbolically $f \prec g$ ) if there exists a Schwarz function $u(z) \in B$ for which $f(z)=g(u(z))$. This result is known as principle of subordination.

By $S^{*}$, we denote the class of starlike functions $f \in S$ which satisfies the following condition:

$$
\operatorname{Re}\left(\frac{z f^{\prime}(z)}{f(z)}\right)>0 \text { or } \frac{z f^{\prime}(z)}{f(z)} \prec \frac{1+z}{1-z},(z \in U) .
$$

By $K$, we denote the class of convex functions $f \in S$ which satisfies the following condition:

$$
\operatorname{Re}\left(\frac{\left(z f^{\prime}(z)\right)^{\prime}}{f^{\prime}(z)}\right)>0 \text { or } \frac{\left(z f^{\prime}(z)\right)^{\prime}}{f^{\prime}(z)} \prec \frac{1+z}{1-z},(z \in U) .
$$

A function $f \in S$ is said to be $\alpha$-convex if it satisfies the inequality

$$
R e\left((1-\alpha) \frac{z f^{\prime}(z)}{f(z)}+\alpha \frac{\left(z f^{\prime}(z)\right)^{\prime}}{f^{\prime}(z)}\right)>0(0 \leq \alpha \leq 1, z \in U) .
$$

The class of $\alpha$-convex functions is denoted by $M(\alpha)$ and was introduced by Mocanu [13]. In particular $M(0) \equiv S^{*}$ and $M(1) \equiv K$.

For $f \in A$, Sãlãgean [17] introduced the following operator:

$$
D^{0} f(z)=f(z), D^{1} f(z)=z f^{\prime}(z),
$$

and in general,

$$
D^{n} f(z)=D\left(D^{n-1} f(z)\right), n \in N
$$

or equivalent to

$$
D^{n} f(z)=z+\sum_{k=2}^{\infty} k^{n} a_{k} z^{k}, n \in N_{0}=N \cup\{0\} .
$$

The inverse functions of the functions in the class $S$ may not be defined on the entire unit disc $U$ although the functions in the class $S$ are invertible. However using Koebe-one quarter theorem [4] it is obvious that the image of $U$ under every function $f \in S$ contains a disc of radius $\frac{1}{4}$. Hence every univalent function $f$ has an inverse $f^{-1}$, defined by

$$
f^{-1}(f(z))=z(z \in U)
$$


and

$$
f\left(f^{-1}(w)\right)=w\left(|w|<r_{0}(f): r_{0}(f) \geq \frac{1}{4}\right)
$$

where

$$
g(w)=f^{-1}(w)=w-a_{2} w^{2}+\left(2 a_{2}^{2}-a_{3}\right) w^{3}-\left(5 a_{2}^{3}-5 a_{2} a_{3}+a_{4}\right) w^{4}+\ldots
$$

A function $f \in A$ is said to be bi-univalent in $U$ if both $f$ and $f^{-1}$ are univalent in $\mathrm{U}$. By $\Sigma$, we denote the class of bi-univalent functions in $U$ defined by (1).

Lewin [10] discussed the class $\Sigma$ of bi-univalent functions and obtained the bound for the second coefficient. Brannan and Taha [1] investigated certain subclasses of bi-univalent functions, similar to the familiar subclasses of univalent functions consisting of strongly starlike, starlike and convex functions. They introduced bi-starlike functions and bi-convex functions and obtained estimates on the initial coefficients.

In 1976, Noonan and Thomas [14] stated the $q$ th Hankel determinant for $q \geq 1$ and $n \geq 1$ as

$$
H_{q}(n)=\left|\begin{array}{cccc}
a_{n} & a_{n+1} & \ldots & a_{n+q+1} \\
a_{n+1} & \ldots & \ldots & \ldots \\
\ldots & \ldots & \ldots & \ldots \\
a_{n+q+1} & \ldots & \ldots & a_{n+2 q-2}
\end{array}\right| .
$$

This determinant has also been considered by several authors. For example, Noor [15] determined the rate of growth of $H_{q}(n)$ as $n \rightarrow \infty$ for functions given by Eq. (1) with bounded boundary. Ehrenborg [5] studied the Hankel determinant of exponential polynomials and the Hankel transform of an integer sequence is defined and some of its properties discussed by Layman [9]. Also Hankel determinant was studied by various authors including Hayman [8], Mehrok and Singh [12], Singh and Singh [19] and Singh [18].

Easily, one can observe that the Fekete-Szegö functional is $H_{2}(1)$. Fekete and Szegö [6] then further generalised the estimate of $\left|a_{3}-\mu a_{2}^{2}\right|$ where $\mu$ is real and $f \in S$. For our discussion in this paper, we consider the Hankel determinant in the case of $q=2$ and $n=2$,

$$
\left|\begin{array}{ll}
a_{2} & a_{3} \\
a_{3} & a_{4}
\end{array}\right|
$$

Sokól [20] introduced the class $S L$ of shell-like functions $f \in A$ defined as below:

Definition 1 A function $f \in A$ given by (1) is said to be in the class $S L$ of starlike shell-like functions if it satisfies the following condition:

$$
\frac{z f^{\prime}(z)}{f(z)} \prec \tilde{p}(z)=\frac{1+\tau^{2} z^{2}}{1-\tau z-\tau^{2} z^{2}}
$$

where $\tau=\frac{1-\sqrt{5}}{2} \approx-0.618$.

It should be observed that $S L$ is a subclass of the class $S^{*}$ of starlike functions. 
Later Dziok et al. [2] introduced the class $K S L$ of convex functions related to a shell-like curve as under:

Definition 2 A function $f \in A$ given by (1) is said to be in the class KSL of convex shell-like functions if it satisfies the condition that

$$
1+\frac{z f^{\prime \prime}(z)}{f^{\prime}(z)} \prec \tilde{p}(z)=\frac{1+\tau^{2} z^{2}}{1-\tau z-\tau^{2} z^{2}}
$$

where $\tau=\frac{1-\sqrt{5}}{2} \approx-0.618$.

Again Dziok et al. [3] defined the following class of $\alpha$-convex shell-like functions:

Definition $3 A$ function $f \in A$ given by (1) is said to be in the class $S L M_{\alpha}$ of $\alpha$-convex shell-like functions if it satisfies the condition that

$$
(1-\alpha) \frac{z f^{\prime}(z)}{f(z)}+\alpha\left(1+\frac{z f^{\prime \prime}(z)}{f^{\prime}(z)}\right) \prec \tilde{p}(z)=\frac{1+\tau^{2} z^{2}}{1-\tau z-\tau^{2} z^{2}}
$$

where $\tau=\frac{1-\sqrt{5}}{2} \approx-0.618$.

Obviously $S L M_{0} \equiv S L$ and $S L M_{1} \equiv K S L$.

Motivated by the above defined classes, we introduce the following subclasses of univalent functions related to shell-like curves connected with Fibinacci numbers as below:

Definition 4 A function $f \in A$ given by (1) is said to be in the class $S L M^{\alpha}$ if it satisfies the condition that

$$
\frac{z f^{\prime}(z)+\alpha z^{2} f^{\prime \prime}(z)}{(1-\alpha) f(z)+\alpha z f^{\prime}(z)} \prec \tilde{p}(z)=\frac{1+\tau^{2} z^{2}}{1-\tau z-\tau^{2} z^{2}}
$$

where $\tau=\frac{1-\sqrt{5}}{2} \approx-0.618$.

Obviously $S L M^{\alpha}$ is a subclass of the class of $\alpha$-convex functions. Also $S L M^{0} \equiv S L$ and $S L M^{1} \equiv K S L$.

Definition 5 A function $f \in A$ given by (1) is said to be in the class $S L M_{\alpha}(n)$ if it satisfies the condition that

$$
(1-\alpha) \frac{D^{n+1} f(z)}{D^{n} f(z)}+\alpha \frac{\left(D^{n+1} f(z)\right)^{\prime}}{\left(D^{n} f(z)\right)^{\prime}} \prec \tilde{p}(z)=\frac{1+\tau^{2} z^{2}}{1-\tau z-\tau^{2} z^{2}}
$$

where $\tau=\frac{1-\sqrt{5}}{2} \approx-0.618$.

We have the following observations:

(i) $S L M_{\alpha}(0) \equiv S L M_{\alpha}$.

(ii) $S L M_{0}(0) \equiv S L$.

(iii) $S L M_{1}(0) \equiv K S L$. 
The function $\tilde{p}$ is not univalent in $U$, but it is univalent in the disc $|z|<\frac{3-\sqrt{5}}{2} \approx$ 0.38. For example, $\tilde{p}(0)=\tilde{p}\left(\frac{-1}{2 \tau}\right)=1$ and $\tilde{p}\left(e^{\mp i \arccos (1 / 4)}\right)=\frac{\sqrt{5}}{5}$, and it may also be noticed that

$$
\frac{1}{|\tau|}=\frac{|\tau|}{1-|\tau|}
$$

which shows that the number $|\tau|$ divides $[0,1]$ such that it fulfils the golden section. The image of the unit circle $|z|=1$ under $\tilde{p}$ is a curve described by the equation given by

$$
(10 x-\sqrt{5}) y^{2}=(\sqrt{5}-2 x)(\sqrt{5} x-1)^{2},
$$

which is translated and revolved trisectrix of Maclaurin. The curve $\tilde{p}\left(r e^{i t}\right)$ is a closed curve without any loops for $0<r \leq r_{0}=\frac{3-\sqrt{5}}{2} \approx 0.38$. For $r_{0}<r<1$, it has a loop, and for $r=1$, it has a vertical asymptote. Since $\tau$ satisfies the equation $\tau^{2}=1+\tau$, this expression can be used to obtain higher powers $\tau^{n}$ as a linear function of lower powers, which in turn can be decomposed all the way down to a linear combination of $\tau$ and 1. The resulting recurrence relationships yield Fibonacci numbers $u_{n}$ :

$$
\tau^{n}=u_{n} \tau+u_{n-1} .
$$

Also the subclasses of bi-univalent functions related to shell-like curves were studied by various authors $[11,21,22]$.

The earlier work on bi-univalent functions related to shell-like curves connected with Fibonacci numbers motivate us to define the following subclasses:

To avoid repetition, throughout the paper we assume that $0 \leq \alpha \leq 1, \tau=$ $\frac{1-\sqrt{5}}{2} \approx-0.618$ and $z \in U$.

Definition 6 A function $f \in \Sigma$ given by (1) is said to be in the class $S L M^{(\alpha, \Sigma)}(\tilde{p}(z))$ if it satisfies the following conditions:

$$
\frac{z f^{\prime}(z)+\alpha z^{2} f^{\prime \prime}(z)}{(1-\alpha) f(z)+\alpha z f^{\prime}(z)} \prec \tilde{p}(z)=\frac{1+\tau^{2} z^{2}}{1-\tau z-\tau^{2} z^{2}}
$$

and

$$
\frac{w g^{\prime}(w)+\alpha w^{2} g^{\prime \prime}(w)}{(1-\alpha) g(w)+\alpha w g^{\prime}(w)} \prec \tilde{p}(w)=\frac{1+\tau^{2} w^{2}}{1-\tau w-\tau^{2} w^{2}}
$$

where $g=f^{-1}$ and $z, w \in U$.

In particular

(i) $S L M^{(0, \Sigma)}(\tilde{p}(z)) \equiv S L_{\Sigma}(\tilde{p}(z))$, the class of bi-univalent starlike shell-like functions studied by Güney et al.[7]].

(ii) $S L M^{(1, \Sigma)}(\tilde{p}(z)) \equiv K S L_{\Sigma}(\tilde{p}(z))$, the class of bi-univalent convex shell-like functions studied by Güney et al.[Y]. 
Definition 7 A function $f \in \Sigma$ given by (1) is said to be in the class $S L M_{\alpha, \Sigma}(n, \tilde{p}(z))$ if it satisfies the following conditions:

$$
(1-\alpha) \frac{D^{n+1} f(z)}{D^{n} f(z)}+\alpha \frac{\left(D^{n+1} f(z)\right)^{\prime}}{\left(D^{n} f(z)\right)^{\prime}} \prec \tilde{p}(z)=\frac{1+\tau^{2} z^{2}}{1-\tau z-\tau^{2} z^{2}}
$$

and

$$
(1-\alpha) \frac{D^{n+1} h(w)}{D^{n} h(w)}+\alpha \frac{\left(D^{n+1} h(w)\right)^{\prime}}{\left(D^{n} h(w)\right)^{\prime}} \prec \tilde{p}(w)=\frac{1+\tau^{2} w^{2}}{1-\tau w-\tau^{2} w^{2}}
$$

where $h=f^{-1}$ and $z, w \in U$.

The following observations are obvious:

(i) $\operatorname{SLM}_{\alpha, \Sigma}(0, \tilde{p}(z)) \equiv S L M_{\alpha, \Sigma}(\tilde{p}(z))$, the class of bi-univalent $\alpha$-convex shell-like functions studied by Güney et al.[7].

(ii) $S L M_{0, \Sigma}(0, \tilde{p}(z)) \equiv S L_{\Sigma}(\tilde{p}(z))$.

(iii) $S L M_{1, \Sigma}(0, \tilde{p}(z)) \equiv K S L_{\Sigma}(\tilde{p}(z))$.

In this paper we inroduce certain subclasses of univalent and bi-univalent functions related to shell-like curves connected with Fibonacci numbers. We find estimates of the initial coefficients $\left|a_{2}\right|$ and $\left|a_{3}\right|$ and upper bounds for the Fekete-Szegö functional and second Hankel determinant for the functions in these classes.

\section{Coefficient bounds for the function classes $S L M^{(\alpha, \Sigma)}(\tilde{p}(z))$ and $S L M_{\alpha, \Sigma}(n, \tilde{p}(z))$}

For deriving our results, we need the following lemma:

Lemma 1 [16] If $p \in P$ be family of all functions $p$ analytic in $U$ for which $\operatorname{Re}[p(z)]>0$ and have the form $p(z)=1+p_{1} z+p_{2} z^{2}+\ldots$ for $z \in U$, then $\left|p_{n}\right| \leq 2$ for each $n$.

Theorem 1 If $f \in S L M^{(\alpha, \Sigma)}(\tilde{p}(z))$, then

$$
\left|a_{2}\right| \leq \frac{|\tau|}{\sqrt{(1+\alpha)^{2}-\left(2+4 \alpha+4 \alpha^{2}\right) \tau}}
$$

and

$$
\left|a_{3}\right| \leq \frac{|\tau|\left[(1+\alpha)^{2}-\left(4+8 \alpha+4 \alpha^{2}\right) \tau\right]}{2(1+2 \alpha)\left[(1+\alpha)^{2}-\left(2+4 \alpha+4 \alpha^{2}\right) \tau\right]}
$$

Proof. As $f \in S L M^{(\alpha, \Sigma)}(\tilde{p}(z))$, so by Definition 6 and using the concept of subordination, there exists Schwarz functions $r(z)$ and $s(z)$ such that

$$
\frac{z f^{\prime}(z)+\alpha z^{2} f^{\prime \prime}(z)}{(1-\alpha) f(z)+\alpha z f^{\prime}(z)}=\tilde{p}(r(z))
$$


and

$$
\frac{w g^{\prime}(w)+\alpha w^{2} g^{\prime \prime}(w)}{(1-\alpha) g(w)+\alpha w g^{\prime}(w)}=\tilde{p}(s(w))
$$

where $r(z)=1+r_{1} z+r_{2} z^{2}+\ldots$ and $s(w)=1+s_{1} w+s_{2} w^{2}+\ldots$

On expanding, it yields

(11) $\frac{z f^{\prime}(z)+\alpha z^{2} f^{\prime \prime}(z)}{(1-\alpha) f(z)+\alpha z f^{\prime}(z)}=1+(1+\alpha) a_{2} z+\left(2(1+2 \alpha) a_{3}-(1+\alpha)^{2} a_{2}^{2}\right) z^{2}+\ldots$

and

(12)

$\frac{w g^{\prime}(w)+\alpha w^{2} g^{\prime \prime}(w)}{(1-\alpha) g(w)+\alpha w g^{\prime}(w)}=1-(1+\alpha) a_{2} w+\left[-2(1+2 \alpha) a_{3}+\left(3(1+2 \alpha)-\alpha^{2}\right) a_{2}^{2}\right] w^{2}+\ldots$

Again

$$
\tilde{p}(r(z))=1+\frac{\tilde{p}_{1} c_{1} z}{2}+\left[\frac{1}{2}\left(c_{2}-\frac{c_{1}^{2}}{2}\right)+\frac{c_{1}^{2}}{4} \tilde{p}_{2}\right] z^{2}+\ldots
$$

and

$$
\tilde{p}(s(z))=1+\frac{\tilde{p}_{1} d_{1} w}{2}+\left[\frac{1}{2}\left(d_{2}-\frac{d_{1}^{2}}{2}\right)+\frac{d_{1}^{2}}{4} \tilde{p}_{2}\right] w^{2}+\ldots
$$

Using (11) and (13) in (9) and equating the coefficients of $z$ and $z^{2}$, we get

$$
(1+\alpha) a_{2}=\frac{c_{1} \tau}{2}
$$

and

$$
2(1+2 \alpha) a_{3}-(1+\alpha)^{2} a_{2}^{2}=\frac{1}{2}\left(c_{2}-\frac{c_{1}^{2}}{2}\right) \tau+\frac{c_{1}^{2}}{4} 3 \tau^{2} .
$$

Again using (12) and (14) in (10) and equating the coefficients of $w$ and $w^{2}$, we get

$$
-(1+\alpha) a_{2}=\frac{d_{1} \tau}{2}
$$

and

$$
-2(1+2 \alpha) a_{3}+\left[3(1+2 \alpha)-\alpha^{2}\right] a_{2}^{2}=\frac{1}{2}\left(d_{2}-\frac{d_{1}^{2}}{2}\right) \tau+\frac{d_{1}^{2}}{4} 3 \tau^{2} .
$$

From (15) and (16), it is clear that

$$
c_{1}=-d_{1}
$$


and

$$
2 a_{2}^{2}=\frac{\left(c_{1}^{2}+d_{1}^{2}\right) \tau^{2}}{4(1+\alpha)^{2}}
$$

Adding (16) and (18), it yields

$$
2\left(1+2 \alpha-\alpha^{2}\right) a_{2}^{2}=\frac{1}{2}\left(c_{2}+d_{2}\right) \tau-\frac{1}{4}\left(c_{1}^{2}+d_{1}^{2}\right) \tau+\frac{3}{4}\left(c_{1}^{2}+d_{1}^{2}\right) \tau^{2} .
$$

Putting (20) in (21), we get:

$$
2\left[(1+\alpha)^{2}+\tau\left(-2-4 \alpha-4 \alpha^{2}\right) a_{2}^{2}=\frac{1}{2}\left(c_{2}+d_{2}\right) \tau^{2} .\right.
$$

Using Lemma 1 and on applying triangle inequality in (22), (7) can be easily obtained.

Now subtracting (18) from (16), we obtain

$$
4(1+2 \alpha) a_{3}-(8 \alpha+4) a_{2}^{2}=\frac{1}{2}\left(c_{2}-d_{2}\right) \tau .
$$

Applying triangle inequality and using Lemma 1 and (22) in (23), it yields

$$
4(1+2 \alpha)\left|a_{3}\right| \leq 2|\tau|+(8 \alpha+4)\left|a_{2}\right|^{2} .
$$

From (24), result (8) is obvious.

For $\alpha=0$, Theorem 1 agrees with the following result proved by Güney et al. $[7]($ Corollary 1$)$ :

Corollary 1 If $f(z) \in S L_{\Sigma}(\tilde{p}(z))$, then

$$
\left|a_{2}\right| \leq \frac{|\tau|}{\sqrt{1-2 \tau}}
$$

and

$$
\left|a_{3}\right| \leq \frac{|\tau|(1-4 \tau)}{2(1-2 \tau)} .
$$

For $\alpha=1$, Theorem 1 agrees with the following result proved by Güney et al.[7] (Corollary 2):

Corollary 2 If $f(z) \in K S L_{\Sigma}(\tilde{p}(z))$, then

$$
\left|a_{2}\right| \leq \frac{|\tau|}{\sqrt{4-10 \tau}}
$$

and

$$
\left|a_{3}\right| \leq \frac{|\tau|(1-4 \tau)}{3(2-5 \tau)}
$$


Theorem 2 If $f \in S L M_{\alpha, \Sigma}(n, \tilde{p}(z))$, then

$$
\left|a_{2}\right| \leq \frac{|\tau|}{\sqrt{4^{n}(1+\alpha)^{2}+\left[2(1+2 \alpha) 3^{n}-\left(3 \alpha^{2}+9 \alpha+4\right) 4^{n}\right] \tau}}
$$

and

$$
\left|a_{3}\right| \leq \frac{|\tau| 4^{n}\left[(1+\alpha)^{2}-\left(3 \alpha^{2}+9 \alpha+4\right) \tau\right]}{2(1+2 \alpha) 3^{n}\left[4^{n}(1+\alpha)^{2}+\left(2(1+2 \alpha) 3^{n}-\left(3 \alpha^{2}+9 \alpha+4\right) 4^{n}\right) \tau\right]} .
$$

Proof. As $f \in S L M_{\alpha, \Sigma}(n, \tilde{p}(z))$, so by Definition 7 and using the concept of subordination, there exists Schwarz functions $r(z)$ and $s(z)$ such that

$$
(1-\alpha) \frac{D^{n+1} f(z)}{D^{n} f(z)}+\alpha \frac{\left(D^{n+1} f(z)\right)^{\prime}}{\left(D^{n} f(z)\right)^{\prime}}=\tilde{p}(r(z))
$$

and

$$
(1-\alpha) \frac{D^{n+1} h(w)}{D^{n} h(w)}+\alpha \frac{\left(D^{n+1} h(w)\right)^{\prime}}{\left(D^{n} h(w)\right)^{\prime}}=\tilde{p}(s(w))
$$

where $r(z)=1+r_{1} z+r_{2} z^{2}+\ldots$ and $s(w)=1+s_{1} w+s_{2} w^{2}+\ldots$.

On expanding, it yields

$(1-\alpha) \frac{D^{n+1} f(z)}{D^{n} f(z)}+\alpha \frac{\left(D^{n+1} f(z)\right)^{\prime}}{\left(D^{n} f(z)\right)^{\prime}}=1+(1+\alpha) 2^{n} a_{2} z+\left(2(1+2 \alpha) 3^{n} a_{3}-(1+3 \alpha) 4^{n} a_{2}^{2}\right) z^{2}+\ldots$

and

(30)

$$
\begin{gathered}
(1-\alpha) \frac{D^{n+1} h(w)}{D^{n} h(w)}+\alpha \frac{\left(D^{n+1} h(w)\right)^{\prime}}{\left(D^{n} h(w)\right)^{\prime}}= \\
1-(1+\alpha) 2^{n} a_{2} w+\left[-2(1+2 \alpha) 3^{n} a_{3}+\left(4(1+2 \alpha) 3^{n}-(1+3 \alpha) 4^{n}\right) a_{2}^{2}\right] w^{2}+\ldots
\end{gathered}
$$

Again

$$
\tilde{p}(r(z))=1+\frac{\tilde{p}_{1} c_{1} z}{2}+\left[\frac{1}{2}\left(c_{2}-\frac{c_{1}^{2}}{2}\right)+\frac{c_{1}^{2}}{4} \tilde{p}_{2}\right] z^{2}+\ldots
$$

and

$$
\tilde{p}(s(z))=1+\frac{\tilde{p}_{1} d_{1} w}{2}+\left[\frac{1}{2}\left(d_{2}-\frac{d_{1}^{2}}{2}\right)+\frac{d_{1}^{2}}{4} \tilde{p}_{2}\right] w^{2}+\ldots
$$

Using (29) and (31) in (27) and equating the coefficients of $z$ and $z^{2}$, we get

$$
(1+\alpha) 2^{n} a_{2}=\frac{c_{1} \tau}{2}
$$


and

$$
2(1+2 \alpha) 3^{n} a_{3}-(1+3 \alpha) 4^{n} a_{2}^{2}=\frac{1}{2}\left(c_{2}-\frac{c_{1}^{2}}{2}\right) \tau+\frac{c_{1}^{2}}{4} 3 \tau^{2} .
$$

Again using (30) and (32) in (28) and equating the coefficients of $w$ and $w^{2}$, we get

$$
-(1+\alpha) 2^{n} a_{2}=\frac{d_{1} \tau}{2}
$$

and

(36) $-2(1+2 \alpha) 3^{n} a_{3}+\left[4(1+2 \alpha) 3^{n}-(1+3 \alpha) 4^{n}\right] a_{2}^{2}=\frac{1}{2}\left(d_{2}-\frac{d_{1}^{2}}{2}\right) \tau+\frac{d_{1}^{2}}{4} 3 \tau^{2}$.

From (33) and (35), it is clear that

$$
c_{1}=-d_{1}
$$

and

$$
2 a_{2}^{2}=\frac{\left(c_{1}^{2}+d_{1}^{2}\right) \tau^{2}}{4^{n+1}(1+\alpha)^{2}} .
$$

Adding (34) and (36), it yields

(39) $\left[4(1+2 \alpha) 3^{n}-2(1+3 \alpha) 4^{n}\right] a_{2}^{2}=\frac{1}{2}\left(c_{2}+d_{2}\right) \tau-\frac{1}{4}\left(c_{1}^{2}+d_{1}^{2}\right) \tau+\frac{3}{4}\left(c_{1}^{2}+d_{1}^{2}\right) \tau^{2}$.

Putting (38) in(39), we get:

$$
2\left[\left(2(1+2 \alpha) 3^{n}-\left(3 \alpha^{2}+9 \alpha+4\right) 4^{n}\right) \tau+4^{n}(1+\alpha)^{2}\right] a_{2}^{2}=\frac{1}{2}\left(c_{2}+d_{2}\right) \tau^{2} .
$$

Using Lemma 1 and on applying triangle inequality in (40), (25) can be easily obtained.

Now subtracting (36) from (34), we obtain

$$
4(1+2 \alpha) 3^{n} a_{3}-4(2 \alpha+1) 3^{n} a_{2}^{2}=\frac{1}{2}\left(c_{2}-d_{2}\right) \tau .
$$

Applying triangle inequality and using Lemma 1 and (40) in (41), it yields

$$
4(1+2 \alpha) 3^{n}\left|a_{3}\right| \leq 2|\tau|+4(2 \alpha+1) 3^{n}\left|a_{2}\right|^{2} .
$$

From (42), result (26) is obvious.

For $n=0$, Theorem 2 gives the following result due to Güney et al.[7]:

Corollary 3 If $f(z) \in S L M_{\alpha, \Sigma}(\tilde{p}(z))$, then

$$
\left|a_{2}\right| \leq \frac{|\tau|}{\sqrt{(1+\alpha)^{2}-(1+\alpha)(2+3 \alpha) \tau}}
$$

and

$$
\left|a_{3}\right| \leq \frac{|\tau|\left[(1+\alpha)^{2}-\left(3 \alpha^{2}+9 \alpha+4\right) \tau\right]}{2(1+2 \alpha)(1+\alpha)[(1+\alpha)-(2+3 \alpha) \tau]} .
$$

Also for $n=0, \alpha=0$, Theorem 2 agrees with Corollary 1 and for $n=0, \alpha=1$, Theorem 2 agrees with Corollary 2. 


\section{Fekete-Szegö inequality for the function classes $S L M^{(\alpha, \Sigma)}(\tilde{p}(z))$ and $S L M_{\alpha, \Sigma}(n, \tilde{p}(z))$}

Theorem 3 Let $f(z) \in S L M^{(\alpha, \Sigma)}(\tilde{p}(z))$, then

$$
\left|a_{3}-\mu a_{2}^{2}\right| \leq\left\{\begin{array}{l}
\frac{|\tau|}{2(1+2 \alpha)},|\mu-1| \leq \frac{\left[(1+\alpha)^{2}+\tau\left(-2-4 \alpha-4 \alpha^{2}\right)\right]}{2(1+2 \alpha)|\tau|} ; \\
\frac{|1-\mu| \tau^{2}}{\left[(1+\alpha)^{2}+\tau\left(-2-4 \alpha-4 \alpha^{2}\right)\right]},|\mu-1| \geq \frac{\left[(1+\alpha)^{2}+\tau\left(-2-4 \alpha-4 \alpha^{2}\right)\right]}{2(1+2 \alpha)|\tau|} .
\end{array}\right.
$$

Proof. From (22) and (23), it yields

$$
a_{3}-\mu a_{2}^{2}=(1-\mu) \frac{\tau^{2}\left(c_{2}+d_{2}\right)}{4\left[(1+\alpha)^{2}+\tau\left(-2-4 \alpha-4 \alpha^{2}\right)\right]}+\frac{\tau\left(c_{2}-d_{2}\right)}{8(1+2 \alpha)} .
$$

(44) can be expressed as

$$
a_{3}-\mu a_{2}^{2}=\left[h(\mu)+\frac{\tau}{8(1+2 \alpha)}\right] c_{2}+\left[h(\mu)-\frac{\tau}{8(1+2 \alpha)}\right] d_{2},
$$

where

$$
h(\mu)=\frac{(1-\mu) \tau^{2}}{4\left[(1+\alpha)^{2}+\tau\left(-2-4 \alpha-4 \alpha^{2}\right)\right]} .
$$

Taking modulus, wo obtain

$$
\left|a_{3}-\mu a_{2}^{2}\right| \leq\left\{\begin{array}{l}
\frac{|\tau|}{2(1+2 \alpha)}, 0 \leq|h(\mu)| \leq \frac{|\tau|}{8(1+2 \alpha)} \\
4|h(\mu)|,|h(\mu)| \geq \frac{|\tau|}{8(1+2 \alpha)}
\end{array}\right.
$$

So result (43) can be easily obtained from (46).

For $\alpha=0$, Theorem 3 agrees with the following result proved by Güney et al.[7] (Corollary 4):

Corollary 4 If $f(z) \in S L_{\Sigma}(\tilde{p}(z))$, then

$$
\left|a_{3}-\mu a_{2}^{2}\right| \leq\left\{\begin{array}{l}
\frac{|\tau|}{2},|\mu-1| \leq \frac{1-2 \tau}{2|\tau|} \\
\frac{|1-\mu| \tau^{2}}{1-2 \tau},|\mu-1| \geq \frac{1-2 \tau}{2|\tau|} .
\end{array}\right.
$$

For $\alpha=1$, Theorem 3 agrees with the following result proved by Güney et al.[7] (Corollary 5): 
Corollary 5 If $f(z) \in K S L_{\Sigma}(\tilde{p}(z))$, then

$$
\left|a_{3}-\mu a_{2}^{2}\right| \leq\left\{\begin{array}{l}
\frac{|\tau|}{6},|\mu-1| \leq \frac{2-5 \tau}{3|\tau|} \\
\frac{|1-\mu| \tau^{2}}{2(2-5 \tau)},|\mu-1| \geq \frac{2-5 \tau}{3|\tau|}
\end{array}\right.
$$

Theorem 4 Let $f(z) \in S L M_{\alpha, \Sigma}(n, \tilde{p}(z))$, then

$$
\left|a_{3}-\mu a_{2}^{2}\right| \leq\left\{\begin{array}{l}
\frac{|\tau|}{2(1+2 \alpha) 3^{n}},|\mu-1| \leq \frac{\left[\left(2(1+2 \alpha) 3^{n}-\left(3 \alpha^{2}+9 \alpha+4\right) 4^{n}\right) \tau+(1+\alpha)^{2} 4^{n}\right]}{2|\tau|(1+2 \alpha) 3^{n}} \\
\frac{|1-\mu| \tau^{2}}{\left[\left(2(1+2 \alpha) 3^{n}-\left(3 \alpha^{2}+9 \alpha+4\right) 4^{n}\right) \tau+(1+\alpha)^{2} 4^{n}\right]} \\
|\mu-1| \geq \frac{\left[\left(2(1+2 \alpha) 3^{n}-\left(3 \alpha^{2}+9 \alpha+4\right) 4^{n}\right) \tau+(1+\alpha)^{2} 4^{n}\right]}{2|\tau|(1+2 \alpha) 3^{n}}
\end{array}\right.
$$

Proof. From (40) and (41), it yields

$a_{3}-\mu a_{2}^{2}=(1-\mu) \frac{\tau^{2}\left(c_{2}+d_{2}\right)}{4\left[\left(2(1+2 \alpha) 3^{n}-\left(3 \alpha^{2}+9 \alpha+4\right) 4^{n}\right) \tau+(1+\alpha)^{2} 4^{n}\right]}+\frac{\tau\left(c_{2}-d_{2}\right)}{8(1+2 \alpha) 3^{n}}$.

(48) can be expressed as

$$
a_{3}-\mu a_{2}^{2}=\left[h(\mu)+\frac{\tau}{8(1+2 \alpha) 3^{n}}\right] c_{2}+\left[h(\mu)-\frac{\tau}{8(1+2 \alpha) 3^{n}}\right] d_{2}
$$

where

$$
h(\mu)=\frac{(1-\mu) \tau^{2}}{4\left[\left(2(1+2 \alpha) 3^{n}-\left(3 \alpha^{2}+9 \alpha+4\right) 4^{n}\right) \tau+(1+\alpha)^{2} 4^{n}\right]} .
$$

Taking modulus, wo obtain

$$
\left|a_{3}-\mu a_{2}^{2}\right| \leq\left\{\begin{array}{l}
\frac{|\tau|}{2(1+2 \alpha) 3^{n}}, 0 \leq|h(\mu)| \leq \frac{|\tau|}{8(1+2 \alpha) 3^{n}} \\
4|h(\mu)|,|h(\mu)| \geq \frac{|\tau|}{8(1+2 \alpha) 3^{n}}
\end{array}\right.
$$

So (47) can be easily obtained from (49).

For $n=0$, Theorem 4 gives the following result due to Güney et al.[7]:

Corollary 6 Let $f(z) \in S L M_{\alpha, \Sigma}(\tilde{p}(z))$, then

$$
\left|a_{3}-\mu a_{2}^{2}\right| \leq\left\{\begin{array}{l}
\frac{|\tau|}{2(1+2 \alpha)},|\mu-1| \leq \frac{(1+\alpha)[(1+\alpha)-(2+3 \alpha) \tau]}{2|\tau|(1+2 \alpha)} \\
\frac{|1-\mu| \tau^{2}}{(1+\alpha)[(1+\alpha)-(2+3 \alpha) \tau]},|\mu-1| \geq \frac{(1+\alpha)[(1+\alpha)-(2+3 \alpha) \tau]}{2|\tau|(1+2 \alpha)}
\end{array}\right.
$$

Also for $n=0, \alpha=0$, Theorem 4 agrees with Corollary 4 and for $n=0, \alpha=1$, Theorem 4 agrees with Corollary 5 . 


\section{Second Hankel determinant for the function classes $S L M^{\alpha}(\tilde{p}(z))$ and $S L M_{\alpha}(n, \tilde{p}(z))$}

For deriving our results, we need the following lemmas:

Lemma 2 [21] If $p(z)=1+p_{1} z+p_{2} z^{2}+\ldots$, and $p \prec \tilde{p}$, then

$$
\left|p_{1}\right| \leq|\tau|
$$

and

$$
\left|p_{2}\right| \leq 3 \tau^{2}
$$

Lemma 3 [21] If $p(z)=1+p_{1} z+p_{2} z^{2}+\ldots$, and $p \prec \tilde{p}$, then

$$
\left|p_{3}\right| \leq 4 \tau^{3} .
$$

Theorem 5 Let $f(z) \in S L M^{\alpha}(\tilde{p}(z))$, then

$$
\left|a_{2} a_{4}-a_{3}^{2}\right| \leq \frac{41 \alpha^{2}+44 \alpha+11}{3(1+\alpha)(1+2 \alpha)^{2}(1+3 \alpha)} \tau^{4} .
$$

Proof. As $f(z) \in S L M^{\alpha}(\tilde{p}(z))$, so by definition 4, we have

$$
\frac{z f^{\prime}(z)+\alpha z^{2} f^{\prime \prime}(z)}{(1-\alpha) f(z)+\alpha z f^{\prime}(z)}=p(z)=1+p_{1} z+p_{2} z^{2}+\ldots
$$

On expanding and equating the coefficients of $z, z^{2}$ and $z^{3}$ in (50), we get

$$
\begin{gathered}
a_{2}=\frac{p_{1}}{1+\alpha}, \\
a_{3}=\frac{p_{2}}{2(1+2 \alpha)}+\frac{p_{1}^{2}}{2(1+2 \alpha)},
\end{gathered}
$$

and

$$
a_{4}=\frac{p_{3}}{3(1+3 \alpha)}+\frac{p_{1} p_{2}}{2(1+3 \alpha)}+\frac{p_{1}^{3}}{6(1+3 \alpha)} .
$$

So, using Lemma 2 and Lemma 3, we obtain

$$
\left|a_{2} a_{4}-a_{3}^{2}\right| \leq \frac{41 \alpha^{2}+44 \alpha+11}{3(1+\alpha)(1+2 \alpha)^{2}(1+3 \alpha)} \tau^{4} .
$$

For $\alpha=0$, Theorem 5 gives the following result due to Sokól et al.[21]:

Corollary 7 If $f(z) \in S L$, then

$$
\left|a_{2} a_{4}-a_{3}^{2}\right| \leq \frac{11}{3} \tau^{4}
$$

For $\alpha=1$, Theorem 5 gives the following result due to Sokól et al.[21]: 
Corollary 8 If $f(z) \in K S L$, then

$$
\left|a_{2} a_{4}-a_{3}^{2}\right| \leq \frac{4}{9} \tau^{4}
$$

Theorem 6 Let $f(z) \in S L M_{\alpha}(n, \tilde{p}(z))$, then

$$
\begin{gathered}
\left|a_{2} a_{4}-a_{3}^{2}\right| \leq \frac{4(1+2 \alpha)^{2}\left(16 \alpha^{2}+19 \alpha+5\right)}{12(1+\alpha)^{4}(1+2 \alpha)^{2}(1+3 \alpha) 8^{n}} \tau^{4} \\
+\frac{3(1+3 \alpha)\left(3 \alpha^{2}+3 \alpha+2\right)^{2} 8^{n}+6(1+2 \alpha)(1+5 \alpha)\left(3 \alpha^{2}+3 \alpha+2\right) 3^{n}}{12(1+\alpha)^{4}(1+2 \alpha)^{2}(1+3 \alpha) 8^{n}} \tau^{4} .
\end{gathered}
$$

Proof. As $f(z) \in S L M_{\alpha}(n, \tilde{p}(z))$, so by definition 5, we have

$$
(1-\alpha) \frac{D^{n+1} f(z)}{D^{n} f(z)}+\alpha \frac{\left(D^{n+1} f(z)\right)^{\prime}}{\left(D^{n} f(z)\right)^{\prime}}=p(z)=1+p_{1} z+p_{2} z^{2}+\ldots
$$

On expanding and equating the coefficients of $z, z^{2}$ and $z^{3}$ in (52), we get

$$
\begin{gathered}
a_{2}=\frac{p_{1}}{2^{n}(1+\alpha)}, \\
a_{3}=\frac{p_{2}}{2(1+2 \alpha)}+\frac{(1+3 \alpha) p_{1}^{2}}{2(1+2 \alpha)(1+\alpha)^{2}},
\end{gathered}
$$

and

$$
\begin{aligned}
& a_{4}=\frac{p_{3}}{3(1+3 \alpha) 4^{n}}+\frac{(1+5 \alpha) 3^{n} p_{1} p_{2}}{2(1+\alpha)(1+2 \alpha)(1+3 \alpha) 4^{n}} \\
& +\frac{\left[(1+3 \alpha)(1+5 \alpha) 3^{n+1}-2(1+2 \alpha)(1+7 \alpha)\right] p_{1}^{3}}{6(1+\alpha)^{3}(1+2 \alpha)(1+3 \alpha) 4^{n}} .
\end{aligned}
$$

So, using Lemma 2 and Lemma 3, we can easily obtain result (51).

For $n=0$, Theorem 6 gives the following result due to Sokól et al.[21]:

Corollary 9 If $f(z) \in S L M_{\alpha}(n)$, then

$$
\left|a_{2} a_{4}-a_{3}^{2}\right| \leq \frac{145 \alpha^{5}+625 \alpha^{4}+1061 \alpha^{3}+867 \alpha^{2}+330 \alpha+44}{12(1+\alpha)^{4}(1+2 \alpha)^{2}(1+3 \alpha)} \tau^{4} .
$$

Also for $n=0, \alpha=0$, Theorem 6 agrees with Corollary 7 and for $n=0, \alpha=1$, Theorem 6 agrees with Corollary 8.

\section{References}

[1] D. A. Brannan, T. S. Taha, On some classes of bi-univalent functions, in: S. M. Mazhar, A. Hamoni, N. S. Faour (Eds.), Mathematical Analysis and its Applications, Kuwait; February 18-21, 1985, in: KFAS Proceedings Series, Vol.3, Pergamon Press, Elsevier Science Limited, Oxford, 1988, pp. 53-60. See also Studia Univ. Babes-Bolyai Math., vol. 31, no. 2, 1986, 70-77. 
[2] J. Dziok, R. K. Raina, J. Sokol, Certain results for a class of convex functions related to a shell-like curve connected with Fibonacci numbers, Comp. Math. Appl., vol. 61, 2011, 2605-2613.

[3] J. Dziok, R. K. Raina, J. Sokol, On $\alpha$-convex functions related to a shell-like curve connected with Fibonacci numbers, Appl. Math. Comp., vol. 218, 2011, 996-1002.

[4] P. L. Duren, Univalent functions, Springer-Verlag, New York, 1983.

[5] R. Ehrenborg, The Hankel determinant of exponential polynomials, American Mathematical Monthly, vol. 107, 2000, 557-560.

[6] M. Fekete, G. Szegö, Eine Bemerkung über ungerade schlichte Funktionen, J. London Math. Soc., vol. 8, 1933, 85-89.

[7] H. Özlem Güney, G. Murugusundaramoorthy, J. Sokól, Subclasses of biunivalent functions related to shell-like curves connected with Fibonacci numbers, Acta Univ. Sapientiae, Mathematica, vol. 10, no. 1, 2018, 70-84.

[8] W. K. Hayman, Multivalent functions, Cambridge Tracts in Math. and Math. Phys., Cambridge University Press, Cambridge, no. 48, 1958.

[9] J. W. Layman, The Hankel transform and some of its properties, J. of Integer Sequences, vol. 4, 2001, 1-11.

[10] M. Lewin, On a coefficient problem for bi-univalent functions, Proc. Amer. Math. Soc., vol. 18, 1967, 63-68.

[11] N. Magesh, V.K.Balaji, C. Abirami, Certain classes of bi-univalent functions related to shell-like curves connected with Fibonacci numbers, Arxiv: 1810.06216V1 [Math CV], 2018.

[12] B. S. Mehrok, G. Singh, Estimate of second Hankel determinant for certain classes of analytic functions, Scientia Magna, vol. 8, no. 3, 2012, 85-94.

[13] P. T. Mocanu, Une propriete'de convexite' généralisée dans la théorie de la représentation conforme, Mathematica(CLUJ), vol. 11, no. 34, 1969, 127-133.

[14] J. W. Noonan, D. K. Thomas, On the second Hankel determinant of a really mean p-valent functions, Trans. Amer. Math. Soc., vol. 223, no. 2, 1976, 337346.

[15] K. I. Noor, Hankel determinant problem for the class of functions with bounded boundary rotation, Rev. Roum. Math. Pures Et Appl., vol. 28, no. 8, 1983, 731-739.

[16] Ch. Pommerenke, Univalent functions, Göttingen: Vandenhoeck and Ruprecht., 1975. 
[17] G. S. Sălăgean, Subclasses of Univalent Functions, Chapter in Lecture Notes in Mathematics, Springer-Verlag, vol. 1013, 1983, 362-372.

[18] G. Singh, Hankel determinant for a new subclass of analytic functions, Scientia Magna, vol. 8, no. 4, 2013, 61-65.

[19] G. Singh, G. Singh, Upper bound of the second Hankel determinant for a subclass of analytic functions, New Trends in Mathematical Sciences, vol. 2, no. 1, 2014, $53-58$.

[20] J. Sokól, On starlike functions connected with Fibonacci numbers, Folia Scient. Univ. Tech. Resoviensis, vol. 175, 1999, 111-116.

[21] J. Sokól, S. Ílhan, Ö. Güney, Second Hankel Determinant problem for several classes of analytic functions related to shell-like curves connected with Fibonacci numbers, TWMS J. App. Eng. Math., vol. 8, no. 1a, 2018, 220-229.

[22] J. Sokól, R. K. Raina, N. Y. Özgür, Applications of K-Fibonacci numbers for the starlike analytic functions, Hacettepe Journal of Math. and Stat., vol. 44, no. 1, 2015, 121-127.

\section{Gurmeet Singh}

G.S.S.D.G.S. Khalsa College

Department of Mathematics

Patiala, Punjab, India.

e-mail: meetgur111@gmail.com

\section{Gagandeep Singh}

Khalsa College

P. G. Department of Mathematics

Amritsar, Punjab, India.

e-mail: kamboj.gagandeep@yahoo.in

\section{Gurcharanjit Singh}

Researh Fellow, Punjabi University

Department of Mathematics

Patiala, Punjab, India.

Assistant Professor, G.N.D.U. College

Department of Mathematics

Chungh(Tarn Taran), Punjab, India.

e-mail: dhillongs82@yahoo.com 